\title{
Are jet ubiquitous in ULXs?
}

\author{
A. Wolter ${ }^{1}$, G. Ghisellini ${ }^{1}$, L. Costamante ${ }^{2}$ and F. Pintore ${ }^{3}$ \\ ${ }^{1}$ INAF-Osservatorio Astronomico di Brera, Milano, Italy; email: anna.wolter@brera.inaf.it \\ ${ }^{2}$ Università di Perugia, Italy, ${ }^{3}$ Università di Cagliari, Italy
}

Abstract. A jet model represents well different X-ray states of the bright ULX IC342 X-1.

Keywords. galaxies: individual (IC 342), X-ray: galaxies, acceleration of particles

\section{Introduction}

Ultra Luminous X-ray sources (ULXs) are point-like, off-nuclear X-ray sources with inferred luminosity (if assumed to be isotropic) in excess of the Eddington limit for spherical accretion onto a $10 \mathrm{M}_{\odot}$ compact object (see e.g., Fabbiano 2006, for a review). IC 342 is a nearby starburst galaxy (3.9 Mpc; Tikhonov \& Galazutdinova 2010) that contains two ULXs: X-1 and X-2, the first ULXs reported to transition between distinct spectral states (Kubota et al. 2001): X-1 went from an apparently disc-dominated state to a power-law-dominated state while the spectrum of X-2 coincidentally carried out the reverse transition. The source X-1 is surrounded by a large optical and radio nebula that may be an X-ray ionized bubble driven by strong outflows from the ULX (Pakull \& Mirioni 2002; Roberts et al. 2003; Feng \& Kaaret 2008). Radio observations of the bubble have been performed with the JVLA at $5 \mathrm{GHz}$ (Cseh et al. 2012): an extended source is detected, with about the same extent of the optical bubble, and a possible unresolved source, albeit not confirmed by Marlowe et al. (2014). The upper limit of the point source is $14 \mathrm{mJy}$ at $5.5 \mathrm{GHz}$.

Could the properties of the ULX X-1 be explained by the presence of a jet?

\section{Results}

We have collected spectral measures at all the available wavelengths in order to construct an overall spectral energy distribution (SED). This SED of IC $342 \mathrm{X}-1$ is plotted in Figure 1: left. In order to test the jet hypothesis we first compare the SED with a known jet source, the Galactic microquasar GRS 1915+105, known to be a superluminal (Mirabel \& Rodriguez 1994) and complexly variable (Fender \& Belloni 2004 for a review) bright source. To mimic this source as pointing towards our line of sight, at an angle of $3^{\circ}$, we boosted the $\mathrm{X}$-ray and radio power by a factor $\delta^{4}$ with $\delta=2.8$. Given the strong agreement we deem that a jet is a viable description of the X-ray emission of this ULX.

We use a model (see Ghisellini \& Tavecchio 2009, for details) that has $M_{\mathrm{BH}}=50 M_{\odot}$ and $L_{\mathrm{Edd}}=9 \times 10^{38} \mathrm{erg} / \mathrm{s}$. The disk luminosity is $L_{\mathrm{disk}}=12 \%$ of $L_{\mathrm{Edd}}$, while the jet, at $100 R_{\mathrm{S}}$, has an angle to the line-of-sight $\theta_{\mathrm{v}}=3^{\circ}$ and $\Gamma_{\text {bulk }}=4$. The magnetic field is $B=2 \times 10^{6} \mathrm{G}$. We include also a corona at $30 \%$ of the disk luminosity and compare it with the SED and in particular with the different X-ray observations from 2004 (see Figure 1: right). The overall X-ray emission is well described by this model in the optical and X-ray energy range; the model never exceeds the upper limit from Fermi in the TeV band. Different X-ray states of IC 342 X-1 can be explained by changing only the injected power (lowest $=30 \%$ of highest) and slightly the electron distribution. 


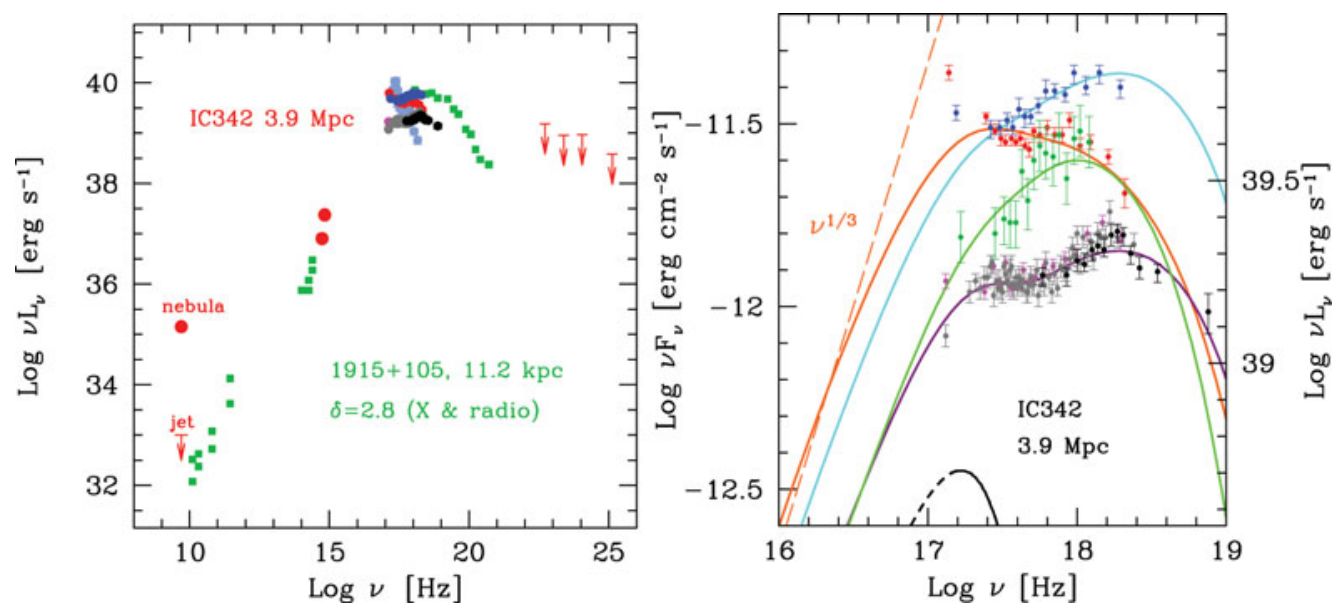

Figure 1. Left: The SED of IC342 X-1 in radio, optical, and $95 \%$ upper limit in the gamma-ray band (red). Fermi data are the upper limit front Aug 2008-Apr 2014 data version ( r $_{-} R E P$; SOURCE class events; Galactic diffuse model v05_rev1; $\Gamma=2$ in each of the 4 bands (Atwood et al. 2009)). The various datasets for XMM-Newton and Swift are represented in pink, blue, pale blue and gray. NuStar data in black. See Pintore et al. (2014); Rana et al. (2014) and references therein. The comparison SED of GRS 1915+105 is shown in green. Right: Zoom of the X-ray band with the models describing different states.

Another Galactic analogue of such a system is the W50 nebula, thought to be inflated by the relativistic jets of the microquasar SS433 (Dubner et al. 1998), which has similar energetic requirements to the IC342 X-1 nebula. Other indications of the presence of jets in ULXs: NGC 2276 3c/S6 (Mezcua et al. 2013; Wolter et al. 2014) with two lobes of radio emission; Holmberg II X-1 (Cseh et al. 2014) with an apparent triple radio structure and attributed to a $\mathrm{BH}$ with $M_{\mathrm{BH}} \geqslant 25 M_{\odot}$. The interpretation with a jet of the X-ray emission is worth of further investigation both with new multi-wavelength observations of this source and the analysis of other ULXs with radio detections.

\section{Acknowledgements}

We acknowledge partial funding from ASI - INAF I/037/12/0.

\section{References}

Atwood, W. B. et al. 2009 ApJ 697, 1071

Cseh, D., Corbel, S., Kaaret, P., et al. 2012 ApJ 749, 17

Cseh, D., Kaaret, P., Corbel, S., et al. 2014 MNRAS 439, L1

Dubner, G. M., Holdaway, M., Goss, W. M., \& Mirabel, I. F. 1998 AJ 116, 1842

Fabbiano, G. 2006, ARAA 44, 323

Feng, H., \& Kaaret, P. 2008 ApJ 675, 1067

Ghisellini, G., \& Tavecchio, F. 2009 MNRAS 397, 985

Kubota, A., Mizuno, T., Makishima, K., et al. 2001 ApJ 547, L119

Marlowe, H., Kaaret, P., Lang, C., et al. 2014 MNRAS 444, 642

Mezcua, M., Roberts, T. P., Sutton, A. D., \& Lobanov, A. P. 2013, MNRAS 436, 3128

Pakull, M. W. \& Mirioni, L. 2002, arXiv:astro-ph/0202488

Pintore F., Zampieri L, Wolter, A., \& Belloni, T. 2014 MNRAS 439, 3461

Rana, V., et al. $2014 A p J$ in press, arXiv:1401.4637

Roberts, T. P., Goad, M. R., Ward, M. J., \& Warwick, R. S. 2003 MNRAS 342, 709

Tikhonov, N. A. \& Galazutdinova, O. A. 2010 Astron. Lett. 36, 167

Wolter, A., Mapelli, M., Esposito, P., Pizzolato, F., \& Ripamonti, E. 2014 MNRAS submitted 